\title{
Dupilumab Decreases Blood Biomarkers in Adolescents With Moderate-to-Severe Atopic Dermatitis: Data From a Phase 3 Trial (LIBERTY AD ADOL)
}

Eric L. Simpson', Hiroyuki Fujita², Kazuhiko Arima², Jennifer Hamilton ${ }^{3}$, Yufang Lư ${ }^{3}$, Ana B. Rossi ${ }^{4}$, Ashish Bansal ${ }^{3}$

'Oregon Health and Science University, Portland, OR, USA; ${ }^{2}$ Sanofi KK, Tokyo, Japan; ${ }^{3}$ Regeneron Pharmaceuticals, Inc., Tarrytown, NY, USA; ${ }^{4}$ Sanofi Genzyme, Cambridge, MA, USA

BACKGROUND

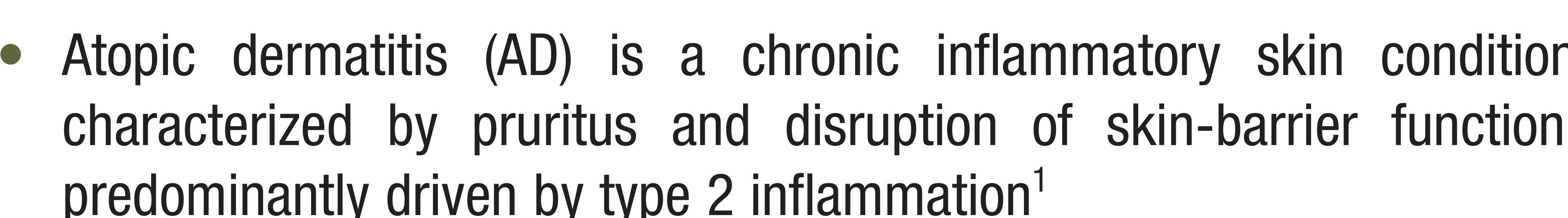

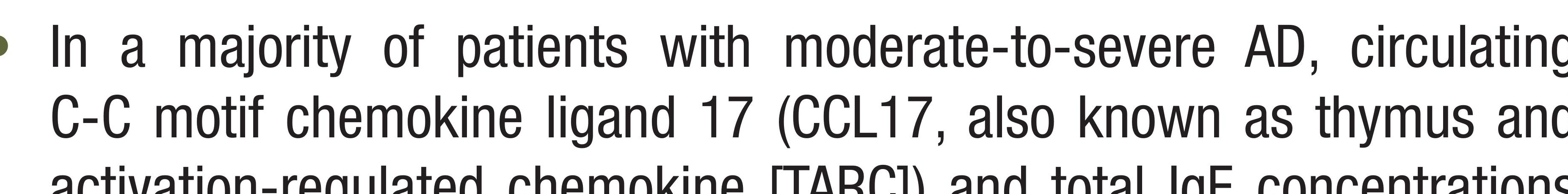

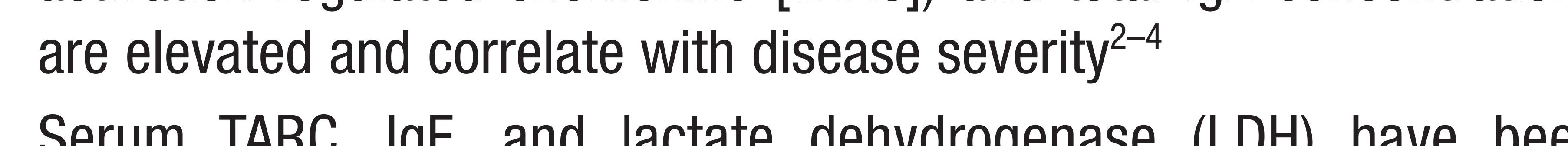

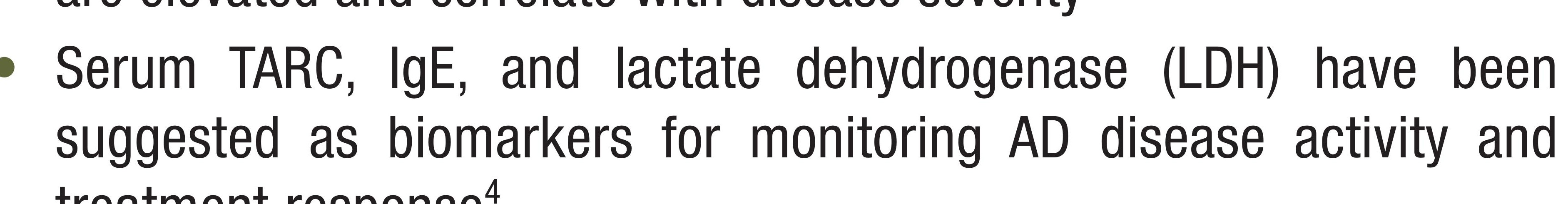

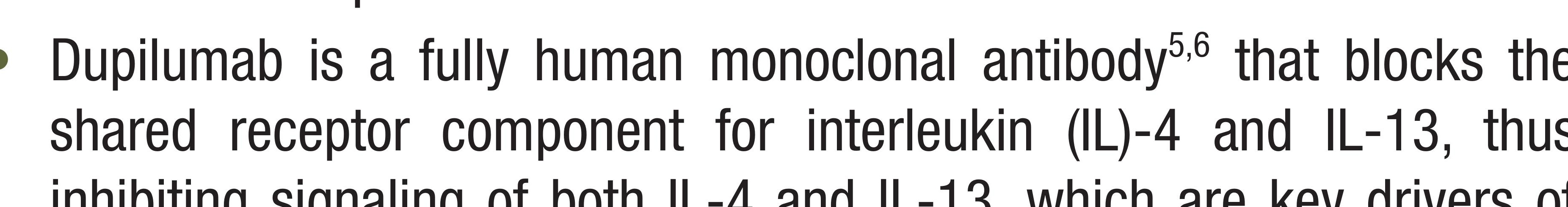

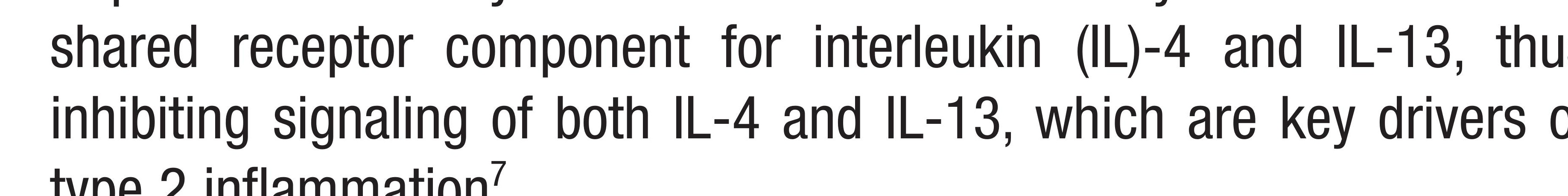

OBJECTIVE

- To evaluate blood levels of type 2 inflammatory

- Markers in patents from a randonzed

placebo-controled, double-blhd, phase 3 thed

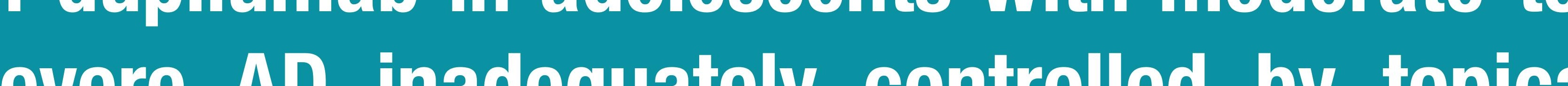

therapies (LIBERTY AD ADOL)

METHODS

tudy design

LIBERTY AD ADOL (NCTO3054428) study desion has been publisted Figure 1. Study design

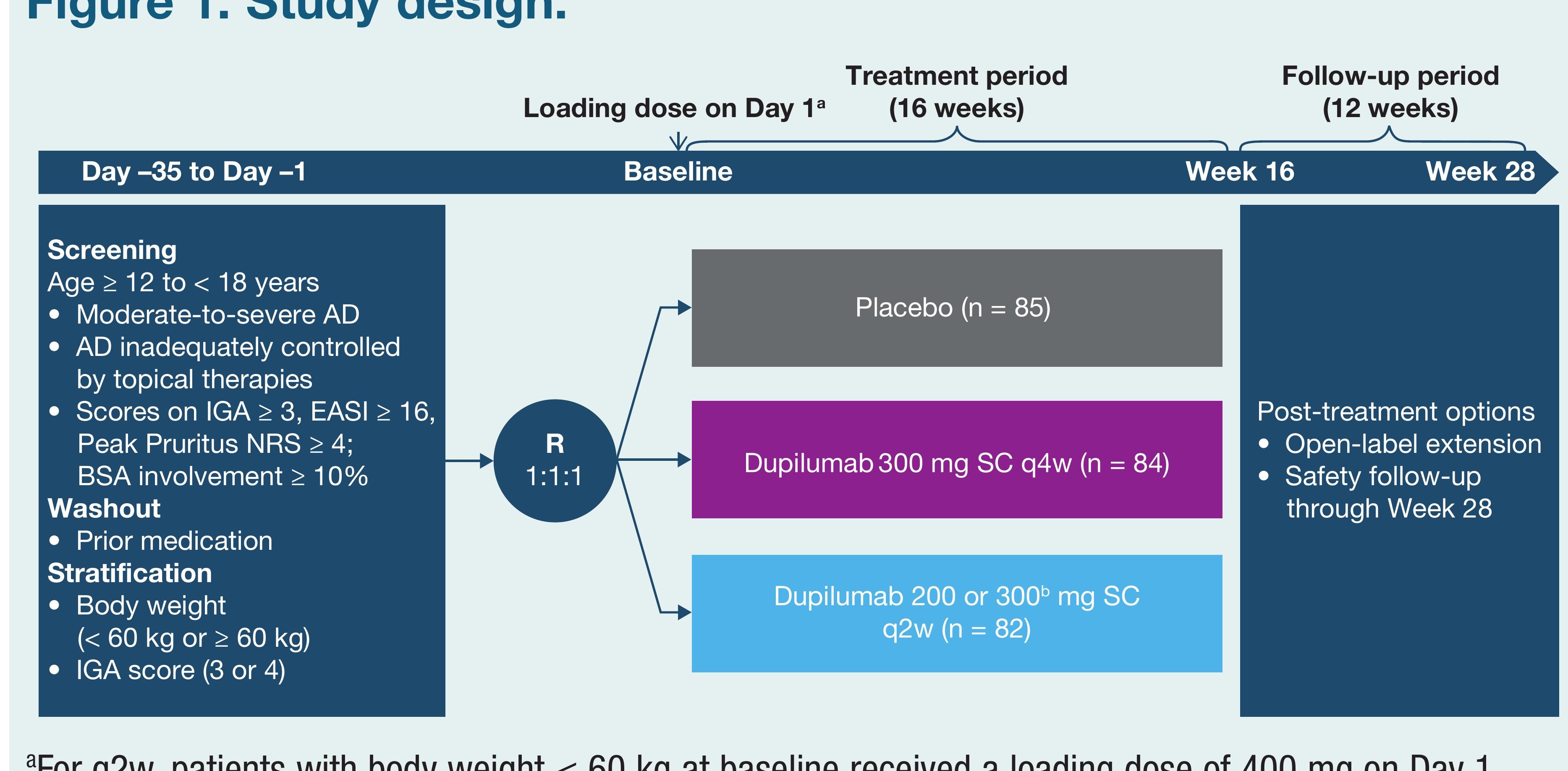

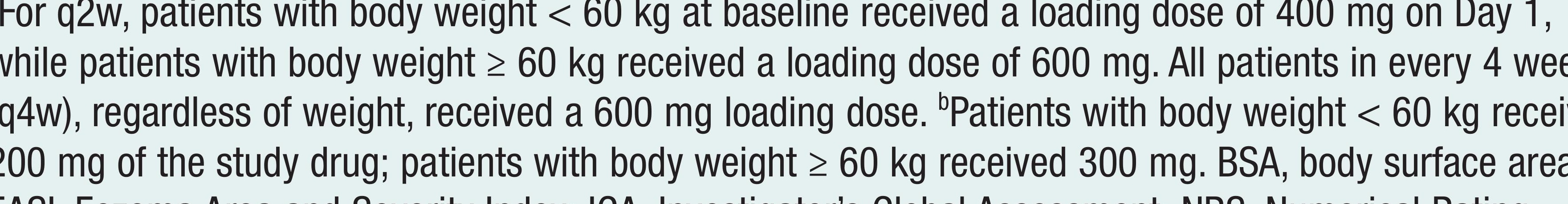

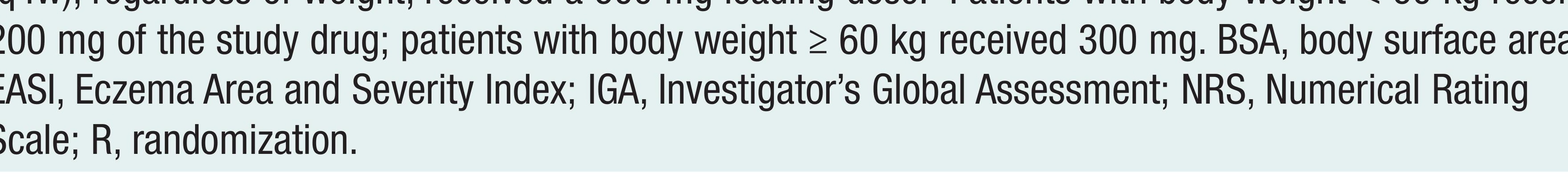


Blood biomarker levels at Week 16

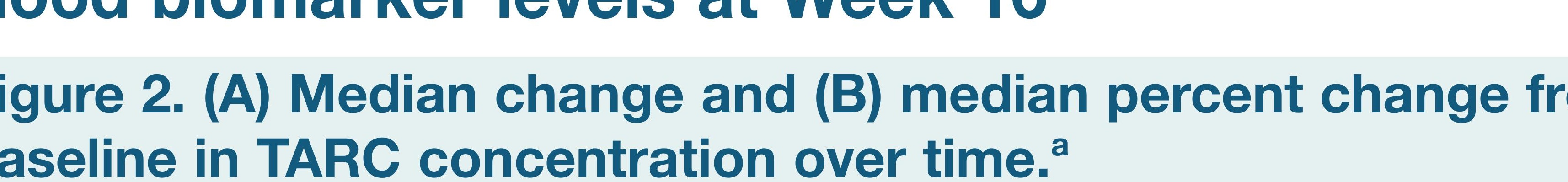
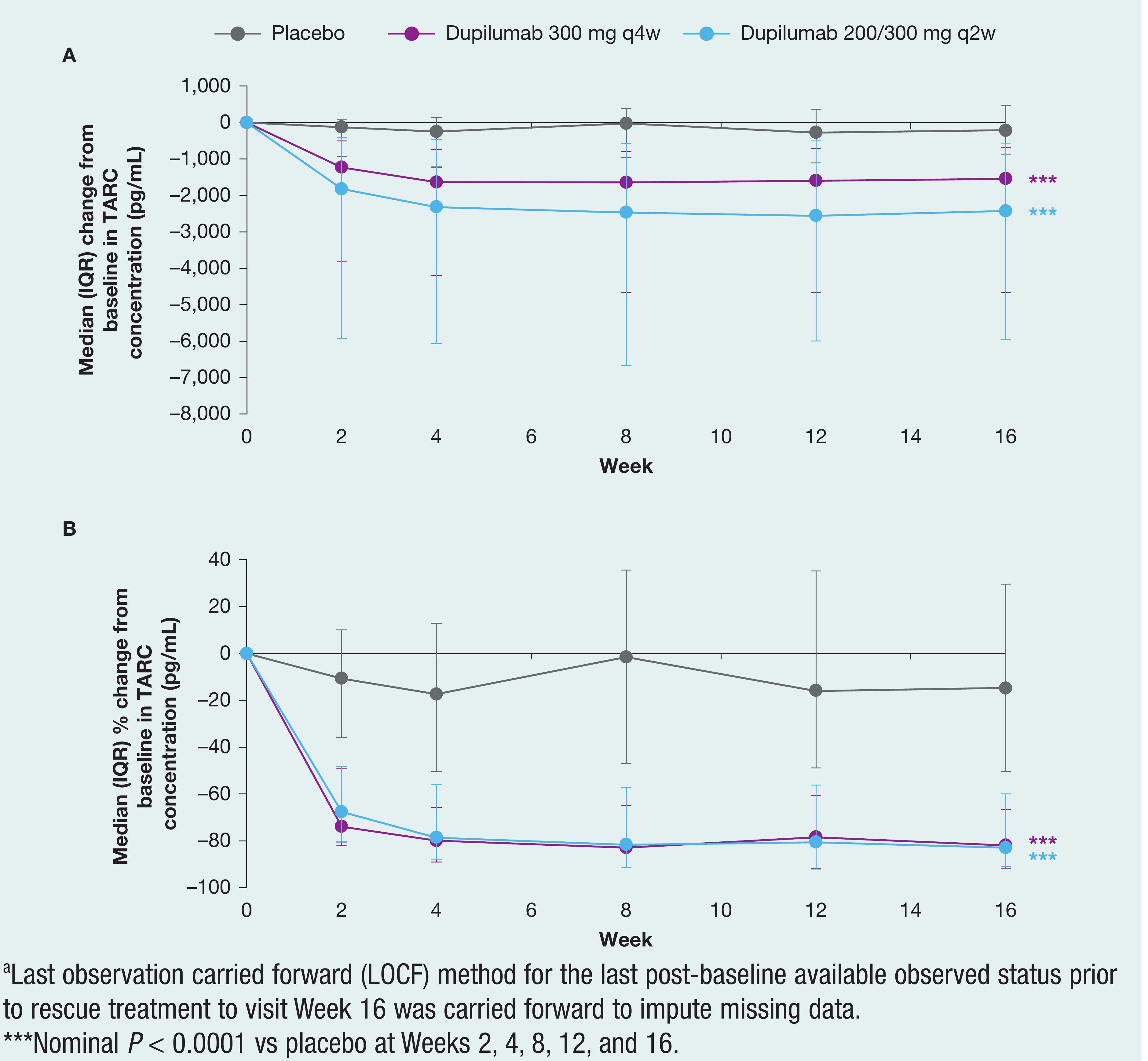

Figure 3. (A) Median change and (B) median percent change fro

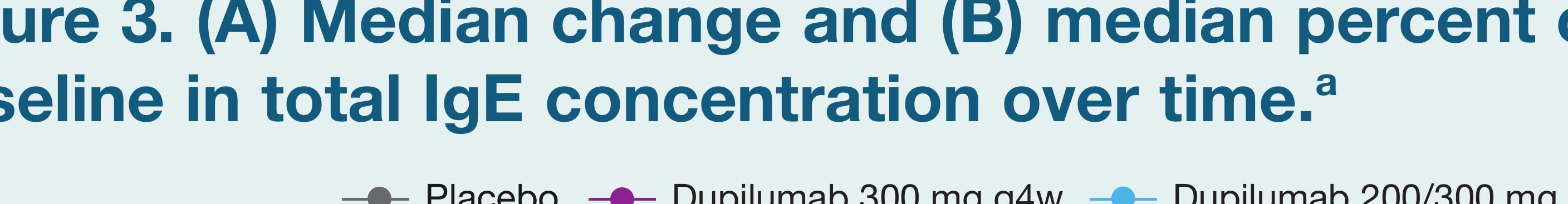

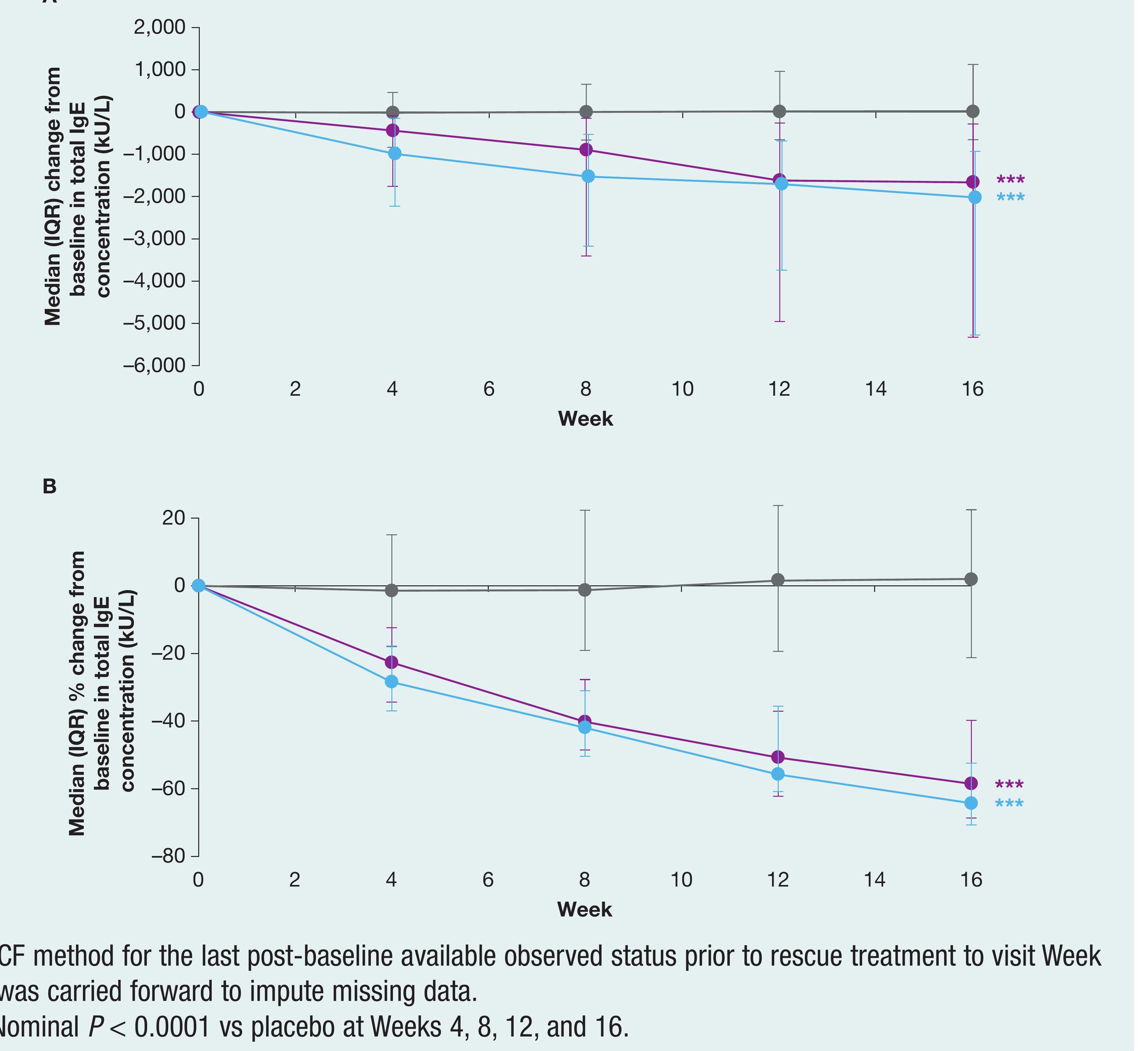

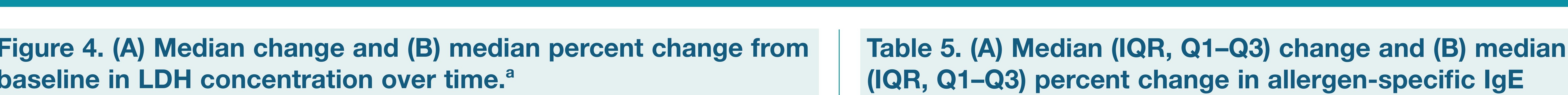

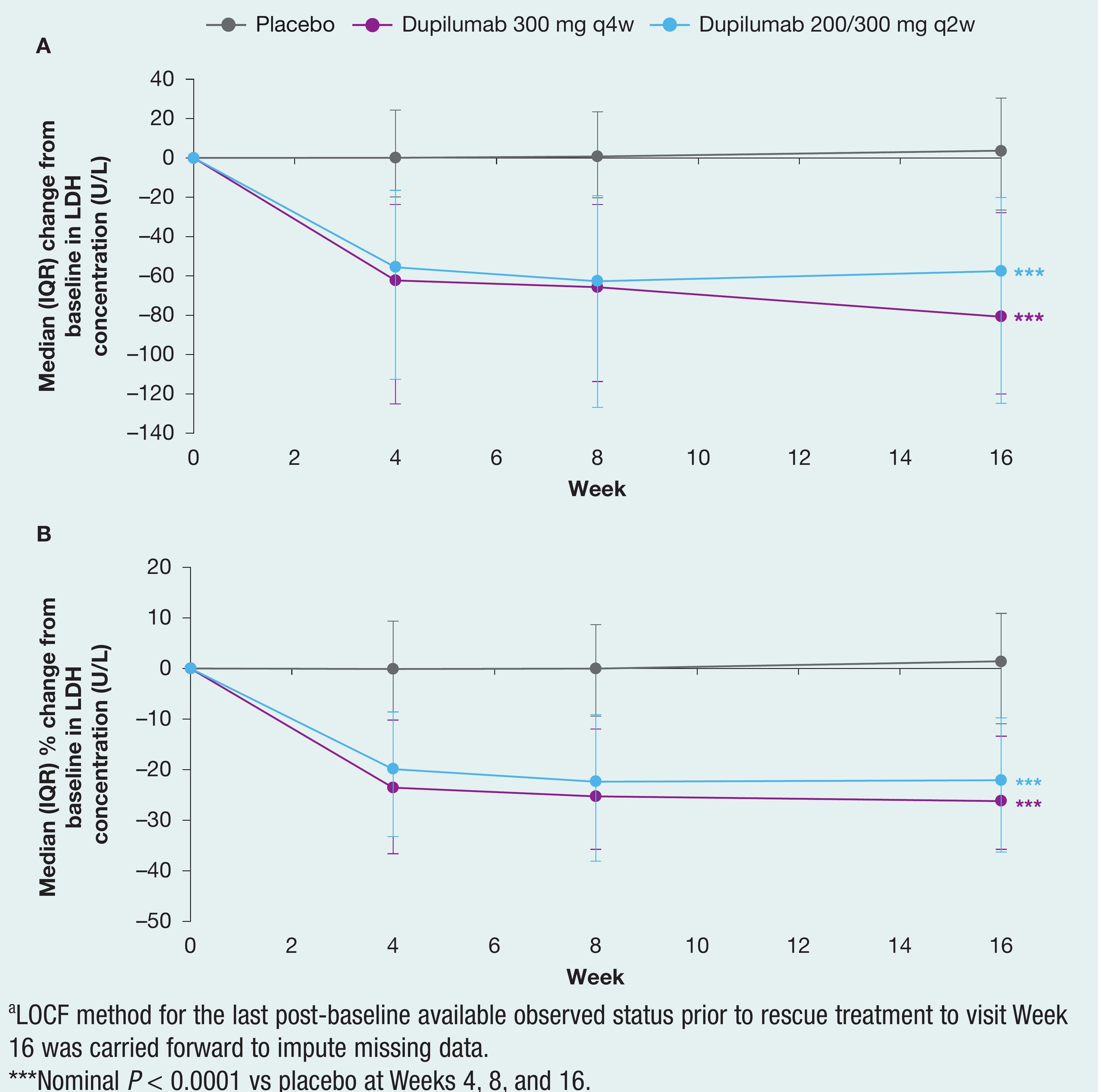

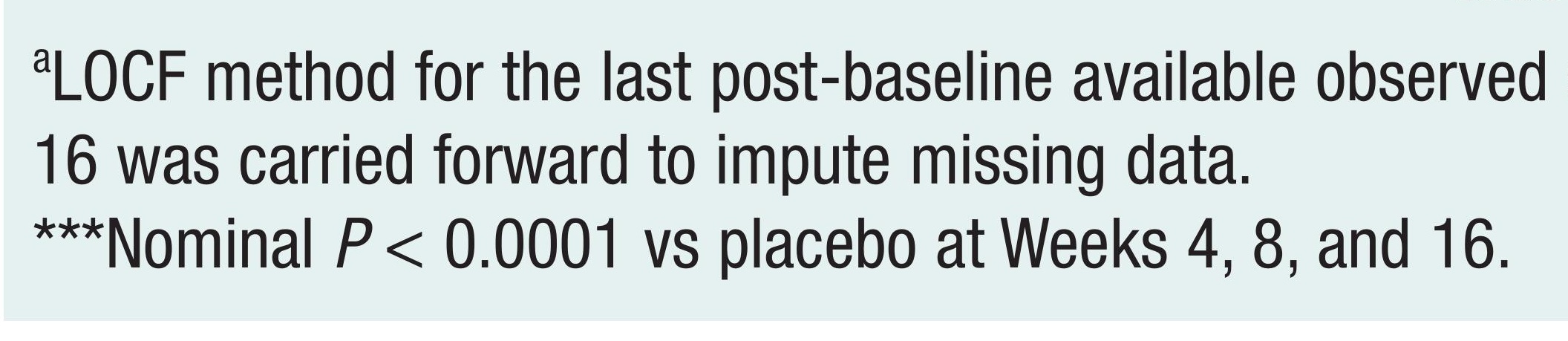
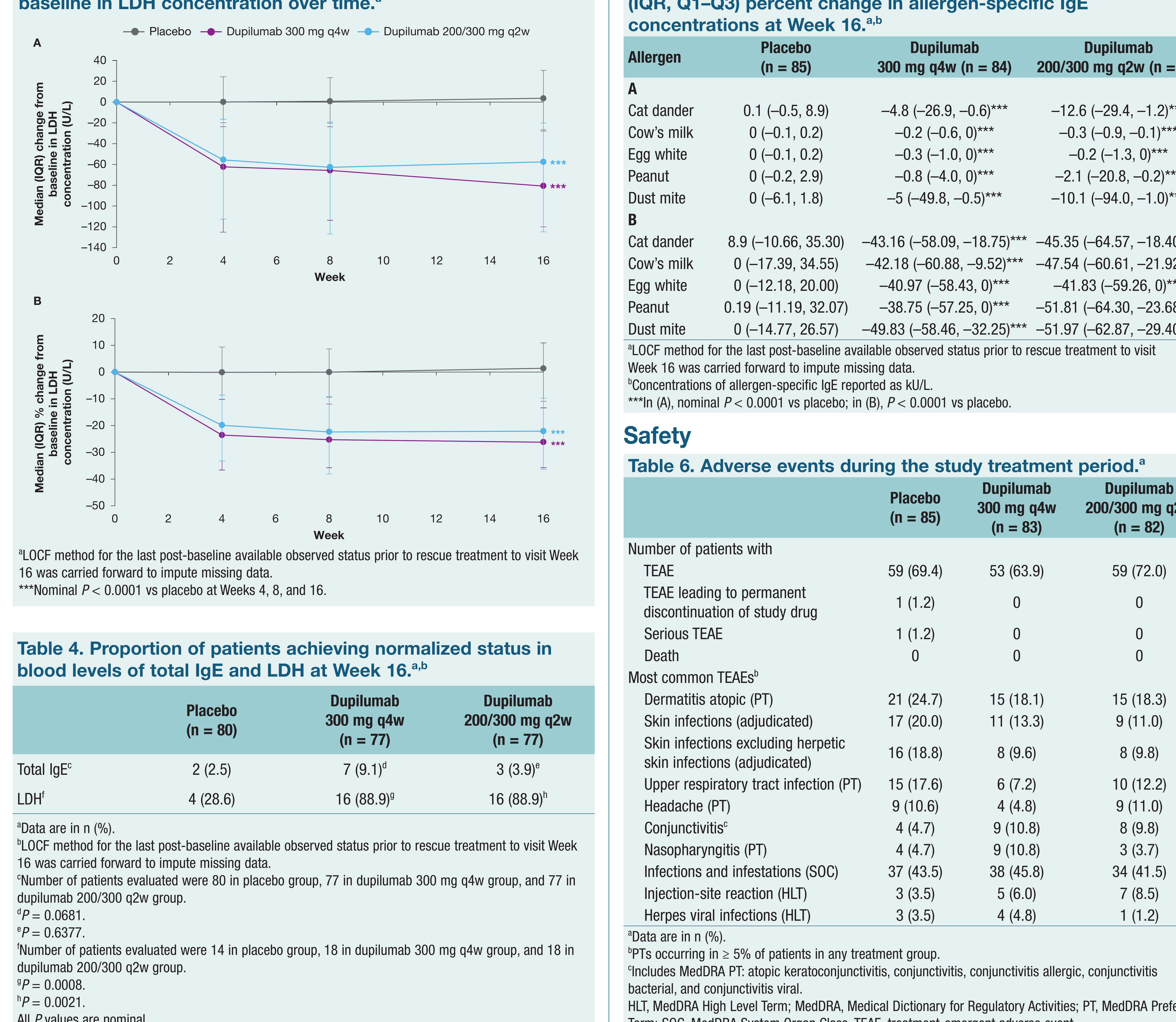

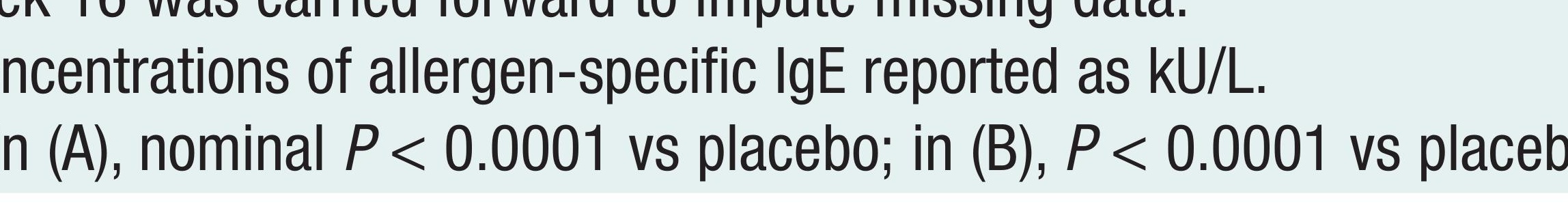

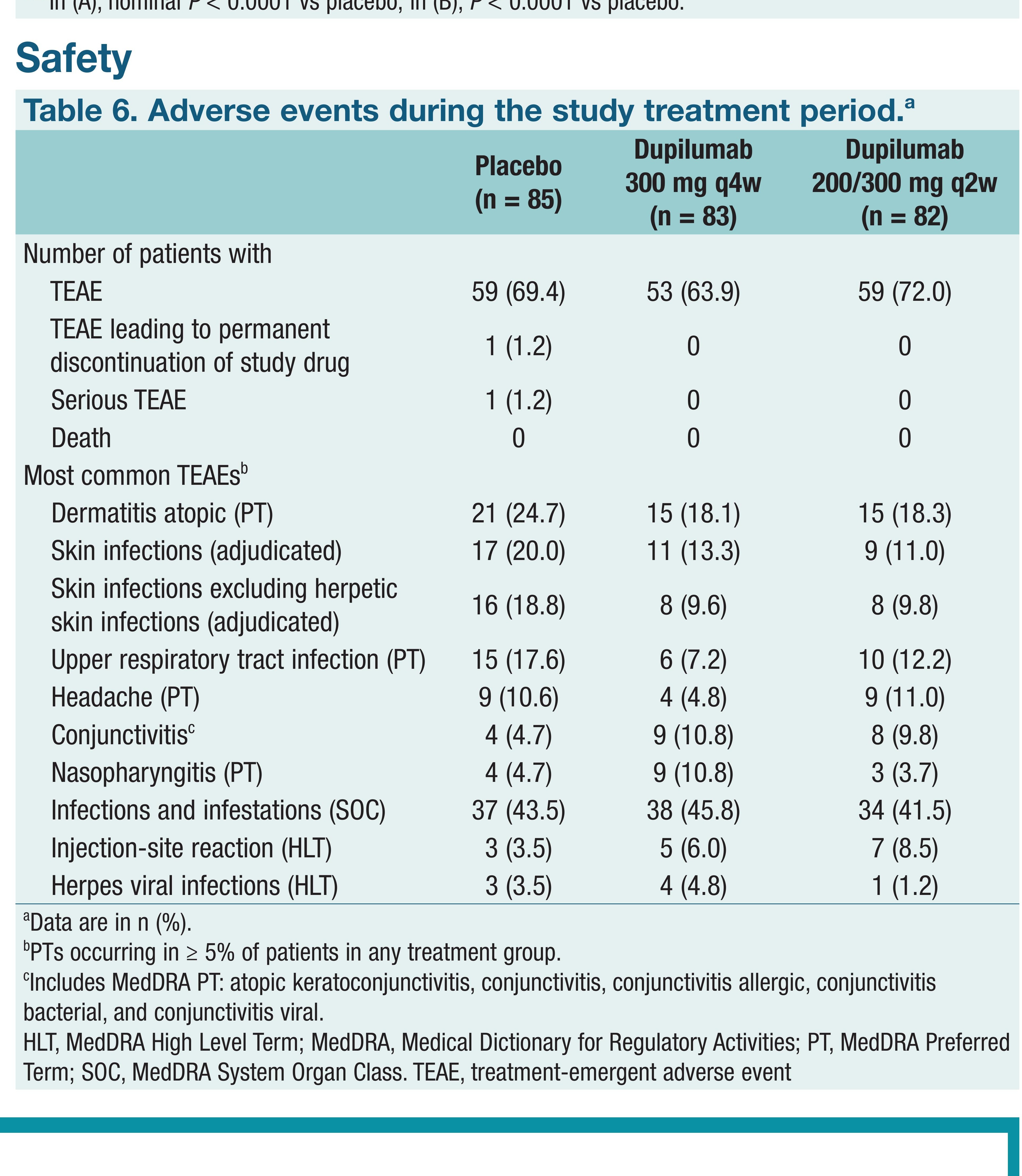

ONCLUSIONS thlaminatory bionarkers (i.e., TARC, total IgE, LDH, and allergen-specific $\operatorname{lgE}$ ); these effects werc accompanied by previously reported improvements in AD signs and symptoms 